\section{A phase II placebo-controlled study of tralokinumab in moderate-to-severe asthma}

\author{
Edward Piper*, Christopher Brightling*, Robert Niven", Chad Oh+, \\ Raffaella Faggioni ${ }^{\S}$, Kwai Poon*, Dewei She ${ }^{+}$, Chris Kell*, Richard D. May*, \\ Gregory P. Geba ${ }^{+}$and Nestor A. Molfino ${ }^{+}$
}

ABSTRACT: Pre-clinical data demonstrate a pivotal role for interleukin (IL)-13 in the development and maintenance of asthma. This study assessed the effects of tralokinumab, an investigational human IL-13-neutralising immunoglobulin G4 monoclonal antibody, in adults with moderate-tosevere uncontrolled asthma despite controller therapies.

194 subjects were randomised to receive tralokinumab $(150,300$ or $600 \mathrm{mg})$ or placebo subcutaneously every 2 weeks. Primary end-point was change from baseline in mean Asthma Control Questionnaire score (ACQ-6; ACQ mean of six individual item scores) at week 13 comparing placebo and combined tralokinumab dose groups. Secondary end-points included pre-bronchodilator lung function, rescue $\beta_{2}$-agonist use and safety. Numerical end-points are reported as mean \pm SD.

At week 13, change from baseline in ACQ-6 was $-0.76 \pm 1.04$ for tralokinumab versus $-0.61 \pm 0.90$ for placebo $(p=0.375)$. Increases from baseline in forced expiratory volume in $1 \mathrm{~s}$ (FEV1) were $0.21 \pm 0.38 \mathrm{~L}$ versus $0.06 \pm 0.48 \mathrm{~L} \quad(p=0.072)$, with a dose-response observed across the tralokinumab doses tested. $\beta_{2}$-agonist use (puffs per day) was decreased for tralokinumab $-0.68 \pm 1.45$ versus placebo $-0.10 \pm 1.49(p=0.020)$. The increase in FEV 1 following tralokinumab treatment remained evident 12 weeks after the final dose. Safety profile was acceptable with no serious adverse events related to tralokinumab.

No improvement in ACQ-6 was observed, although tralokinumab treatment was associated with improved lung function.

KEYWORDS: Asthma, CAT-354, interleukin-13, lung function, monoclonal antibody, tralokinumab

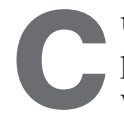
urrent therapy is inadequate in many patients with moderate-to-severe asthma, with a substantial proportion remaining uncontrolled, resulting in an increased risk of exacerbation, increased morbidity and mortality, reduced quality of life, increased direct and indirect healthcare costs and lost productivity [1-4].

Interleukin (IL)-13 is a pleiotropic cytokine believed to be an important mediator in the development and maintenance of the human asthmatic phenotype through its role in key underlying mechanisms including inflammation $[5,6]$, airway hyperresponsiveness (AHR) $[5,6]$, fibrosis [7] and increased mucus production [5, 6]. Elevated IL-13 levels have been identified in the sputum [8] of a proportion of subjects with asthma including those with severe disease treated with systemic corticosteroids.
Tralokinumab (CAT-354) is a human immunoglobulin (Ig)G4 monoclonal antibody which potently and specifically neutralises IL-13 [9] and has been shown to inhibit a range of IL-13-mediated effects in pre-clinical studies [10]. Tralokinumab is in clinical development for the treatment of asthma; phase I studies [11] have demonstrated linear pharmacokinetics and an acceptable safety profile over the dose range tested.

The primary objective of this proof-of-concept study was to evaluate the effect of subcutaneous tralokinumab on asthma control in adults with moderate-to-severe uncontrolled asthma despite standard controller therapy.

\section{METHODS}

\section{Study design}

This was a phase IIa, randomised, double-blind, placebo-controlled, parallel-group, multicentre
AFFILIATIONS

*Medlmmune Ltd, Cambridge,

\# Institute of Lung Health, University

of Leicester, Leicester, and

- University of Manchester and

University Hospital of South

Manchester, Manchester, UK.

+Medlmmune, LLC, Gaithersburg,

$\mathrm{MD}$, and

${ }^{\S}$ Medlmmune, LLC, Hayward, CA, USA.

CORRESPONDENCE

E. Piper

Clinical Development

Medlmmune Ltd

Milstein Building

Granta Park

Cambridge

CB21 6GH

UK

E-mail: pipered@medimmune.com

Received:

Dec 202011

Accepted after revision:

May 082012

First published online:

June 272012

This article was modified in April 2016 to correct errors in the licence information. 
study (Clinicaltrials.gov identifier: NCT00873860). The study comprised a 2-week run-in, 12-week dosing and a 12-week follow-up period. Selection of dose regimen and treatment duration was based on pharmacokinetic modelling and simulations.

\section{Randomisation and masking}

Subjects were stratified prior to randomisation to ensure $\geqslant 50 \%$ of randomised subjects were atopic. The presence or absence of specific IgE to a panel of inhaled allergens determined atopic status (Phadiatop test; Laboratoire Pasteur Cerba, Paris, France). Subjects were then randomised according to a computergenerated randomisation list into one of three cohorts; within each cohort, subjects were further randomised to receive tralokinumab or placebo in a 3:1 ratio. Subjects receiving tralokinumab were administered doses of 150, 300 or $600 \mathrm{mg}$. Treatment was administered every 2 weeks by s.c. injection. Subjects continued their pre-study asthma controller therapy during the study.

\section{Subjects}

Subjects aged 18-65 yrs with body mass index $18-40 \mathrm{~kg} \cdot \mathrm{m}^{-2}$ and physician-diagnosed, moderate-to-severe uncontrolled asthma were eligible and were required to have all of the following: reversible airflow obstruction (post-bronchodilator forced expiratory volume in $1 \mathrm{~s}$ (FEV1) reversibility $\geqslant 12 \%$ and $\geqslant 200 \mathrm{~mL}$ either documented within the previous year or at screening), pre-bronchodilator $\mathrm{FEV} 1 \geqslant 40 \%$ predicted value, Asthma Control Questionnaire score (ACQ-6; ACQ mean of six individual item scores) $\geqslant 1.5$ at screening and randomisation, and one or more asthma exacerbations that required medical intervention in the past year.

Key exclusion criteria were additional respiratory pathology, cigarette smoking $\geqslant 10$ pack-yrs, recent infection or treatment with immunosuppressive medication $(>10 \mathrm{mg}$ oral prednisone or equivalent per day) or any other biologic agent.

This study was conducted in accordance with the principles of the Declaration of Helsinki and the International Conference on Harmonisation Guidance for Good Clinical Practice. Independent ethics committee approval was obtained, and all subjects provided written informed consent.

\section{Primary end-point}

The ACQ-6 [12] is a composite measure of asthma symptoms (night-time waking, symptoms on waking, activity limitation, shortness of breath, wheezing) and short-acting $\beta_{2}$-agonist use which is completed weekly using an electronic diary. The primary end-point of the study was the change from baseline to week 13 in mean ACQ-6 score [12]. Reductions in mean ACQ-6 score of $\geqslant 0.5$ are considered clinically meaningful [13].

\section{Secondary end-points}

Secondary end-points included the evaluation of the effect of tralokinumab compared to placebo on time to asthma control, change from baseline in FEV1 and peak expiratory flow (PEF) (at study visit and at home), time to first asthma exacerbation, asthma exacerbation rate, requirement for concomitant asthma rescue medications, daily asthma symptoms scores and Asthma Quality of Life Questionnaire with standardised activities (AQLQ(S)). Spirometry (pre-bronchodilator FEV1, forced vital capacity (FVC) and PEF) was performed at 06:0011:30 $\mathrm{h}$ at every study visit and within $1 \mathrm{~h}$ of the time that screening visit spirometry had been undertaken; pre-bronchodilator FEV1, FVC and PEF were measured. Post-bronchodilator measurements were not recorded during the treatment phase. Subjects performed peak flow testing every morning at home. Asthma exacerbations were reported at each study visit and were defined as either a progressive increase of asthma symptoms (cough, wheeze, chest tightness and/or shortness of breath) or a reduction of $>20 \%$ in PEF or FEV1 from baseline that did not resolve after the initiation of rescue medications and resulted in an administration of systemic corticosteroids by the investigator or healthcare provider. Patient-reported outcomes (PROs) included a four-item daily asthma symptom score and AQLQ(S).

Adverse events (AEs) were recorded at every study visit; routine blood tests were collected periodically for safety evaluation.

Sputum was induced and collected at baseline and weeks 8 and 14 at nine of the participating study centres. Sputum IL-13 content was assessed using an ELISA system, and differential cell counts were recorded by a central reader (Institute for Lung Health, Leicester, UK).

\section{Pharmacokinetics and immunogenicity}

For pharmacokinetic assessment blood samples were collected prior to each dose and during the follow-up period. Trough serum concentrations of tralokinumab were determined using a Gyrolab immunoassay (Gyros AB, Uppsala, Sweden) that detects free tralokinumab. Blood samples were collected before the first dose and at weeks 13 and 24 for measurement of serum anti-drug antibodies.

\section{Statistical methods}

Sample size calculations based on the primary end-point were conducted using statistical software nQuery Advisor ${ }^{\circledR} 6.01$ (Statistical Solutions, Cork, Ireland). Change from baseline in mean ACQ-6 score at week 13 was assumed to be -0.5 and -1.0 for the placebo and combined tralokinumab (150, 300 and $600 \mathrm{mg}$ ) groups, respectively, with a common SD of 0.9. A sample size of 144 was required to detect a statistically significant difference between combined tralokinumab groups and placebo at a 0.05 significance level with $80 \%$ power. To allow for dropouts, randomisation of 192 subjects was planned.

The efficacy analyses reported are from the evaluable population, defined as all subjects who received $\geqslant 4$ doses of study medication and those who received $\geqslant 1$ dose but discontinued prior to receiving 4 doses for safety reasons. Confirmatory efficacy analysis was undertaken on the intention-to-treat population, defined as all randomised subjects.

The primary end-point was the comparison of the change from baseline to week 13 in mean ACQ-6 score between the combined tralokinumab group (i.e. 150, 300 and $600 \mathrm{mg}$ ) and placebo using ANOVA. The primary efficacy analysis was undertaken at an interim analysis after all subjects completed week 13 although both study team and sites remained blinded to week 24. Two-sample unpaired t-tests were used to compare between treatment group changes from baseline in spirometry parameters. Exploratory efficacy analyses to compare each 
tralokinumab dose with placebo were undertaken and no multiple comparison adjustment was performed.

A number of post hoc analyses were conducted, including analysis of the change from baseline to week 13 in FEV1 in subgroups defined by atopic status, dose of corticosteroid and baseline peripheral eosinophil count, and also the change from baseline to week 13 in FEV1 in subgroups defined by atopic status. A descriptive post hoc analysis of the change from baseline in ACQ-6 and FEV1 was undertaken in a subgroup of subjects with sputum samples obtained at baseline. Subjects were analysed according to the presence or absence of detectable sputum IL-13; no formal statistical testing was undertaken due to the relatively small subject numbers.

Safety analysis was conducted using the safety population, defined as all subjects who received $\geqslant 1$ dose of study medication.

\section{RESULTS}

194 subjects were randomised at 27 sites in Europe (fig. 1). All seven doses of study medication were received by $96 \%$ placebo and $94 \%$ tralokinumab subjects.

\section{Subject characteristics}

Across the study population, $60 \%$ were female, $92 \%$ were white, and age ranged from 18 to 65 yrs (mean 47.3 yrs). At baseline, the study population had moderate-to-severe asthma that was not fully controlled (FEV1 $2.00 \mathrm{~L}$ (61\% pred); ACQ-6 score 2.66). $52 \%$ were atopic as defined above. A range of inhaled corticosteroid (ICS) preparations were taken at baseline; the median daily ICS dose, expressed as beclometasone diproprionate equivalent, was $1,000 \mu \mathrm{g} \cdot \mathrm{day}^{-1}$. $87 \%$ reported use of a longacting $\beta_{2}$-agonist and $20 \%$ a leukotriene antagonist. Baseline demographic and asthma characteristics were generally well balanced across the treatment groups (table 1).

\section{Efficacy}

Primary end-point: change in ACQ-6 score from baseline to week 13

Mean ACQ-6 score improved from baseline to week 13 in all treatment groups (table 2 and fig. 2a), with 58.3\% tralokinumab and $52.2 \%$ placebo subjects showing a clinically meaningful improvement of $\geqslant 0.5$. The mean $\pm S D$ reduction in the combined tralokinumab group $(-0.76 \pm 1.04)$ at week 13 was not different from placebo $(-0.61 \pm 0.90, p=0.375)$. The difference ( $95 \%$ CI) between treatment groups in change from baseline was -0.15 (-0.49-0.19). Reductions in ACQ-6 score continued through week 24 in both groups. The change from baseline in ACQ-6 score at week 13 was examined by tertiles of ACQ score at baseline, atopic status, peripheral blood eosinophil count and dose of asthma controller medication, but no definite relationship to response was identified. Changes in ACQ-6 score in subjects providing a sputum sample at baseline, stratified by the presence or absence of IL-13, are presented in figure $3 \mathrm{a}$. The week 13 mean $\pm \mathrm{SD}$ reduction in the sputum IL13-positive $\left(\geqslant 10 \mathrm{pg} \cdot \mathrm{mL}^{-1}\right)$ tralokinumab group $(-0.97 \pm 0.98$, $n=11)$ was greater than placebo $(-0.43 \pm 0.70, n=17)$ or sputum

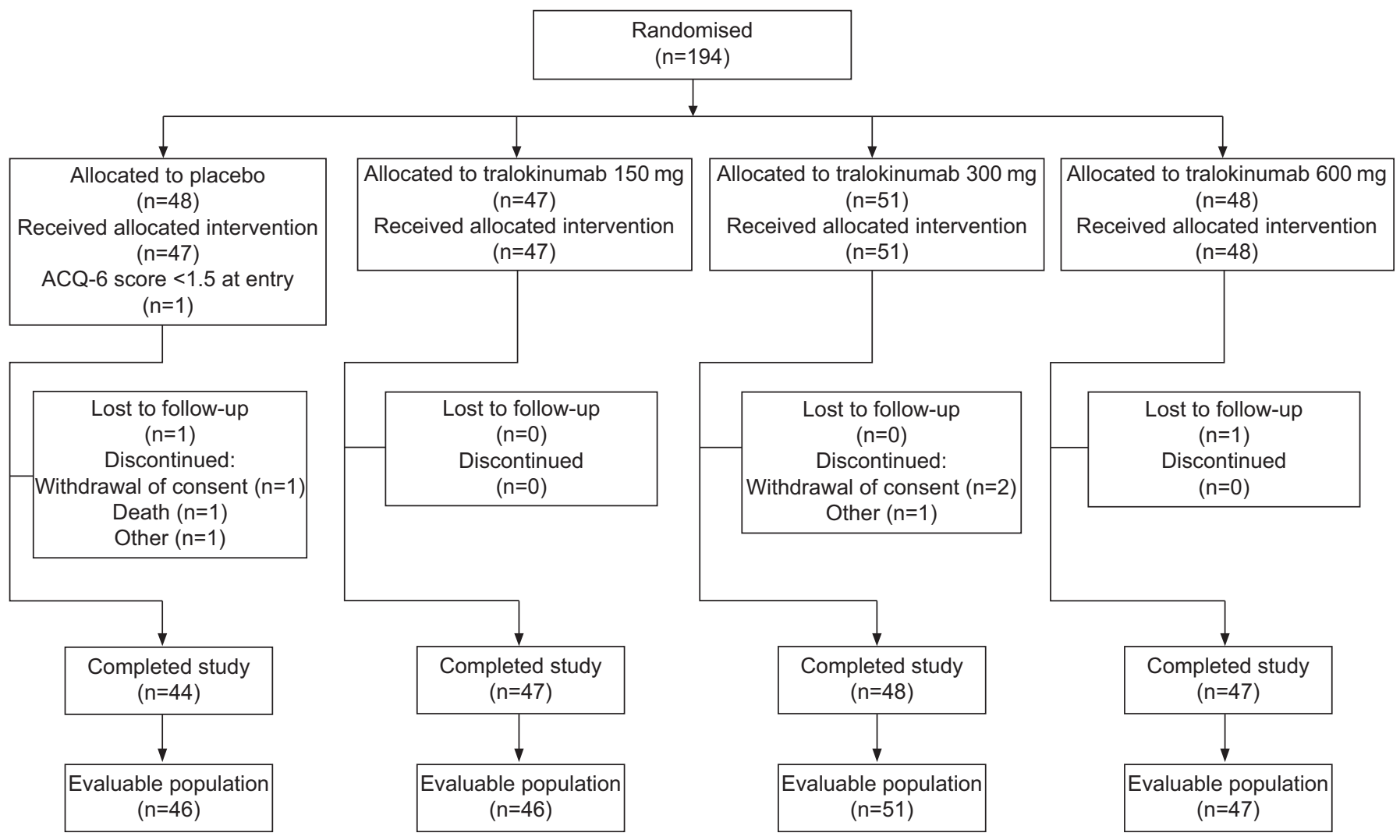

FIGURE 1. Subject disposition. Evaluable population comprised subjects who received at least four doses of study medication. Subjects who received at least one dose but discontinued prior to receiving four doses due to safety reasons were also included in the evaluable population. ACQ-6: Asthma Control Questionnaire, mean of six individual item scores. 
TABLE 1 Baseline subject and disease characteristics: intention-to-treat population

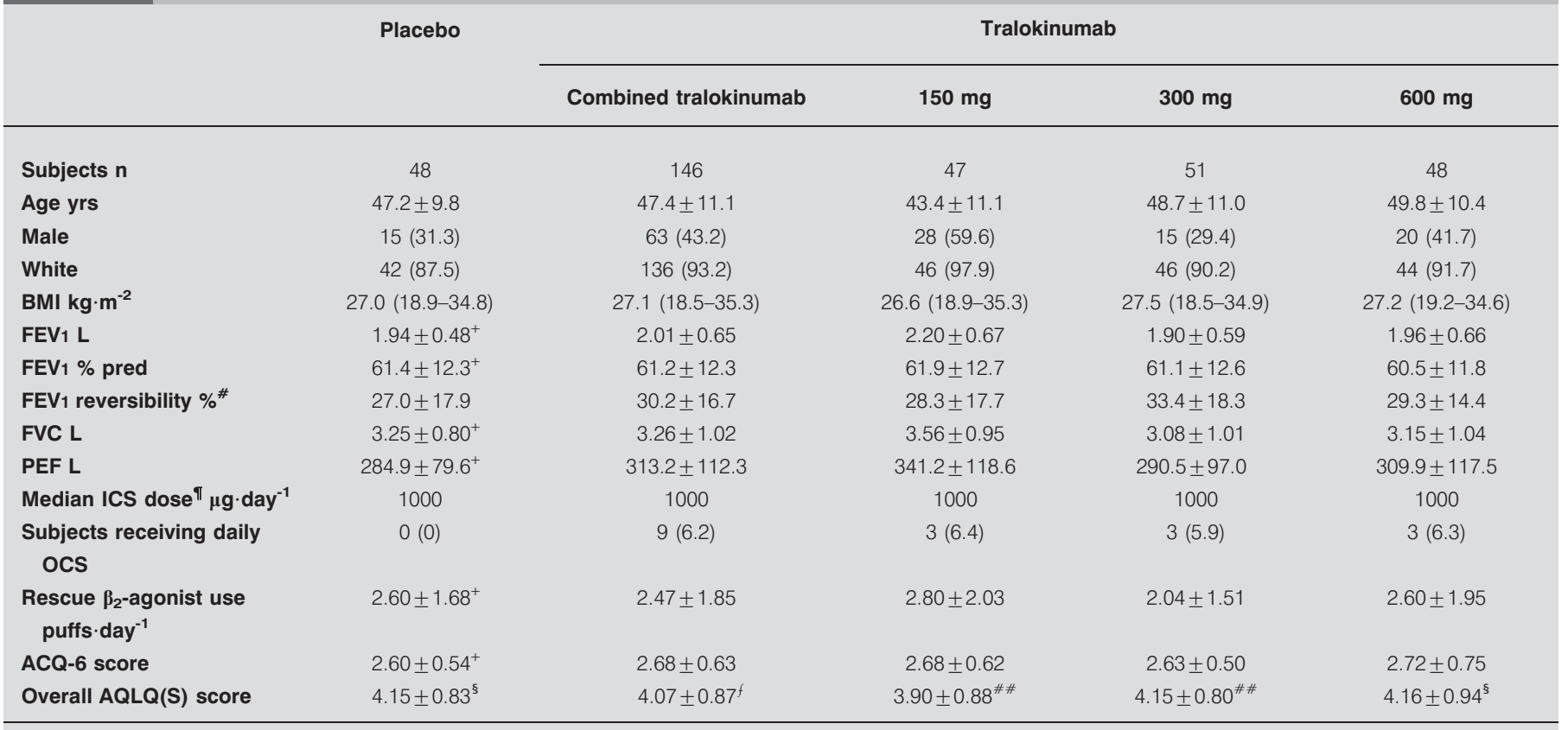

Data are presented as mean \pm SD, $n(\%)$ or mean (range), unless otherwise stated. BMI: body mass index; FEV1: forced expiratory volume in $1 \mathrm{~s} ; \%$ pred: \% predicted; FVC: forced vital capacity; PEF: peak expiratory flow; ICS: inhaled corticosteroids; OCS: oral corticosteroids; ACQ-6: Asthma Control Questionnaire, mean of six individual item scores; AQLQ(S): Asthma Quality of Life Questionnaire with standardised activities. * : mean reversibility of subjects who demonstrated reversibility during screening (excluding those who reported historical reversibility); ${ }^{\bullet}$ : beclometasone equivalent; ${ }^{+}: n=47 ;{ }^{\varsigma}: n=36 ;{ }^{f}: n=114 ; \# \#$ : $n=39$.

IL-13-negative $\left(<10 \mathrm{pg} \cdot \mathrm{mL}^{-1}\right)$ tralokinumab group $(-0.62 \pm 0.85$, $\mathrm{n}=28)$.

\section{Secondary end-points}

Pulmonary function

A larger mean $\pm \mathrm{SD}$ increase in pre-bronchodilator FEV1 from baseline to week 13 was observed in the combined tralokinumab group $(0.21 \pm 0.38 \mathrm{~L})$ compared to placebo $(0.06 \pm 0.48 \mathrm{~L} ; \mathrm{p}=0.072)$; the difference $(95 \% \mathrm{CI})$ in change from baseline was $-0.15 \mathrm{~L}(-0.01-0.31)$. A clear dose-response was evident at the same time-point with mean \pm SD increases from baseline in FEV1 of $0.16 \pm 0.35 \mathrm{~L} \quad(\mathrm{p}=0.299), 0.21 \pm 0.37 \mathrm{~L}$ $(p=0.102)$ and $0.26 \pm 0.41 \mathrm{~L}(p=0.041)$ in the 150,300 , and $600 \mathrm{mg}$ tralokinumab treatment groups, respectively (table 2). The effect on FEV1 was evident 2 weeks after the first dose and persisted until week 24, 12 weeks after the final dose (fig. 2b). Post hoc subgroup analyses showed that similar mean \pm SD increases in FEV1 were observed at week 13 in subjects receiving tralokinumab with atopic $(0.22 \pm 0.39 \mathrm{~L} ; \mathrm{n}=73)$ and non-atopic asthma $(0.20 \pm 0.37 \mathrm{~L} ; \mathrm{n}=64)$ and in those receiving high-dose ICS or oral corticosteroids $(0.19 \pm 0.44 \mathrm{~L} ; \mathrm{n}=46)$. In subjects with peripheral blood eosinophil count $\geqslant 300$ cells $\cdot \mathrm{mm}^{-3}$ at baseline, the increases in FEV1 were $0.26 \pm 0.41 \mathrm{~L}(n=70)$ and in those below this threshold were $0.15 \pm 0.34 \mathrm{~L}(\mathrm{n}=67)$. Changes in FEV1 in subjects providing a sputum sample at baseline, stratified by the presence or absence of IL-13, are presented in figure $3 b$. The week 13 mean \pm SD improvement in the sputum IL-13-positive $\left(\geqslant 10 \mathrm{pg} \cdot \mathrm{mL}^{-1}\right)$ tralokinumab group $(0.37 \pm 0.49 \mathrm{~L} ; \mathrm{n}=11)$ was greater than placebo $(0.12 \pm 0.32 \mathrm{~L} ; \mathrm{n}=15)$ or sputum $\mathrm{IL}-13$ negative $\left(<10 \mathrm{pg} \cdot \mathrm{mL}^{-1}\right)$ tralokinumab group $(0.10 \pm 0.29 \mathrm{~L} ; \mathrm{n}=26)$.
FVC and PEF measured by spirometry at study visits also improved in the combined tralokinumab group compared with placebo, although the changes were not statistically significant (table 2).

Tralokinumab had no statistically significant or clinically relevant effect on home measurements of FEV1 and PEF. The mean \pm SD per cent change in home-measured FEV1 and PEF over the 7 days prior to week 13 was $4.8 \pm 21.8 \%$ and $1.1 \pm 19.5 \%$, respectively, in the combined tralokinumab group and $0.8 \pm 19.5 \%$ and $-1.3 \pm 17.6 \%$, respectively, for placebo.

\section{Use of rescue medication}

Subjects in the combined tralokinumab group showed a significantly greater mean \pm SD reduction in $\beta_{2}$-agonist use compared with placebo at week $13\left(-0.68 \pm 1.45\right.$ versus $-0.10 \pm 1.49$ puffs $\cdot$ day $^{-1}$; $\mathrm{p}=0.020)$ and week $24\left(0.73 \pm 1.57\right.$ versus $-0.05 \pm 1.87$ puffs $\cdot$ day $^{-1}$; $\mathrm{p}=0.025)$.

\section{Asthma exacerbations}

Few subjects experienced asthma exacerbations, and there was no difference between treatment groups. At week 13, protocoldefined asthma exacerbations were reported by four $(2.9 \%)$ tralokinumab and two (4.5\%) placebo subjects.

\section{Patient-reported outcomes}

No differential effect of tralokinumab compared to placebo was apparent on any of the PROs.

\section{Safety and tolerability}

Serious AEs were reported by three subjects receiving tralokinumab and three receiving placebo (one death occurred in the 


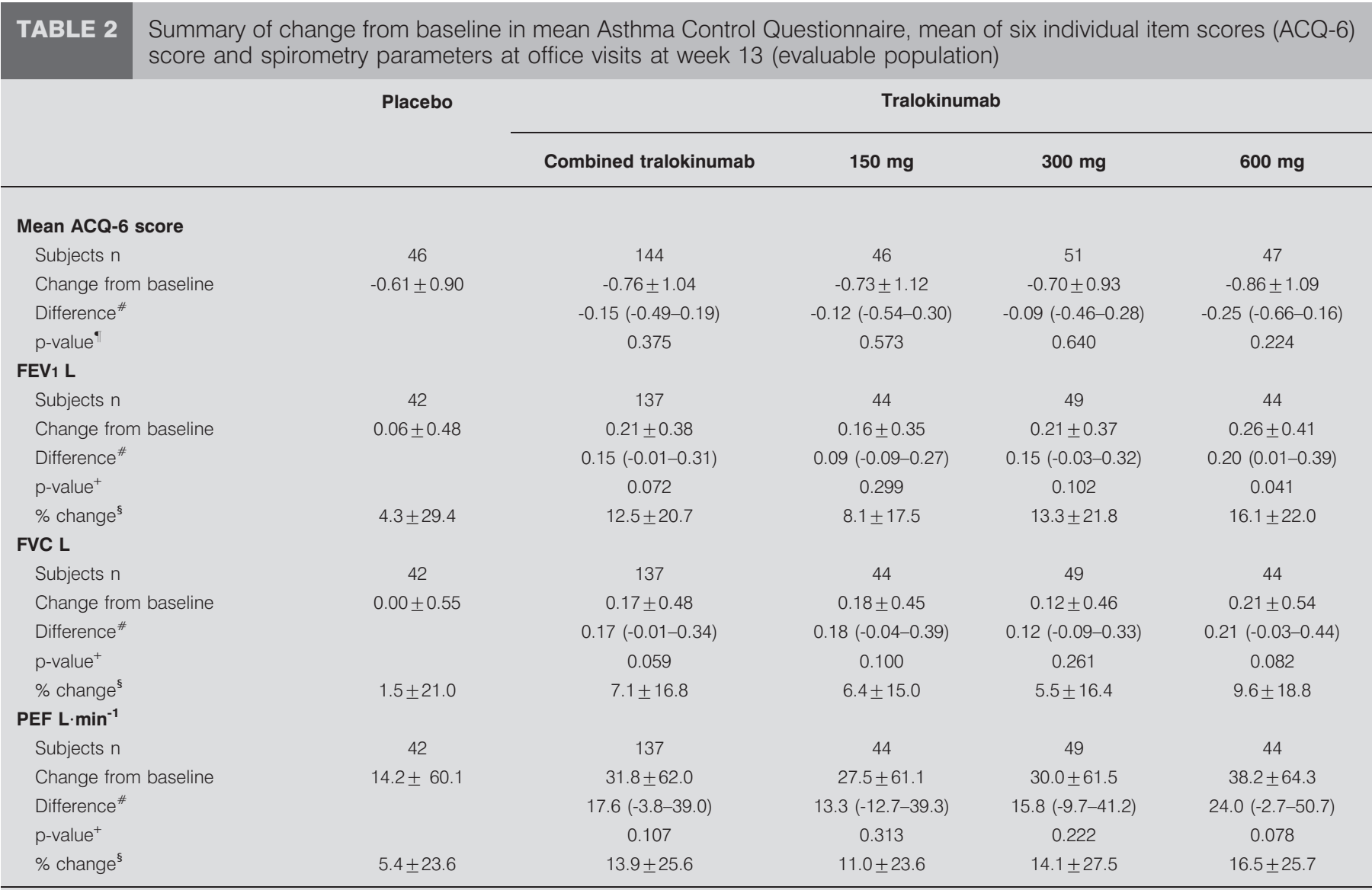

Data are presented as mean \pm SD or mean $(95 \% \mathrm{CI})$, unless otherwise stated. FEV1: forced expiratory volume in $1 \mathrm{~s}$; FVC: forced vital capacity; PEF: peak expiratory flow.

${ }^{\#}$ : difference in mean change from baseline: tralokinumab - placebo; ${ }^{\prime}$ : based on ANOVA; ${ }^{+}$: calculated using a two-sample t-test; ${ }^{\S}$ : change from baseline: current visit value - baseline value.

placebo group). The serious AEs were: sinusitis (tralokinumab $150 \mathrm{mg}$ ); contusion and brain injury following a traffic accident (tralokinumab $150 \mathrm{mg}$ ); gastro-oesophageal reflux disease, post-cholecystectomy syndrome, somatoform disorder (tralokinumab $600 \mathrm{mg}$ ); asthma exacerbation (placebo); asthma exacerbation, overdose of salbutamol, fatal cardiorespiratory arrest (placebo); and cerebral haemorrhage (placebo). None of the serious AEs in subjects who received tralokinumab were considered to be related to study medication.

The incidence of treatment-emergent AEs was higher in the tralokinumab groups than in the placebo group (table 3). The most frequently reported AEs in subjects who received tralokinumab were asthma, headache and nasopharyngitis. More subjects receiving tralokinumab reported an AE of asthma compared with placebo; these were all categorised by investigators as mild or moderate in intensity, whereas two events in the placebo group were severe. Of the AEs of asthma in subjects receiving tralokinumab, two were judged to have a possible relationship to the study drug and approximately one-third occurred during the off-treatment follow-up period.

Diarrhoea and urinary-related AEs (urinary tract infection, bacteriuria, crystalluria) were reported only in subjects who received tralokinumab (table 3); seven subjects with urinary-related AEs received antibiotic treatment. Diarrhoea was limited to subjects in the highest dose arm of tralokinumab.

Injection site reactions (ISRs), comprising of at least one of pain, erythema, rash, pruritus, inflammation and induration occurred infrequently (tralokinumab $150 \mathrm{mg} \mathrm{6.4 \% ;} 300 \mathrm{mg} \mathrm{7.8 \% ;} 600 \mathrm{mg}$ $6.3 \%$; placebo $2.1 \%$ ). Most ISRs were mild and transient; one tralokinumab subject withdrew as a result of an ISR.

There were no clinically important changes in serum chemistry among tralokinumab-treated subjects, whilst the only change noted in haematology parameters was an increase from baseline in mean \pm SD peripheral blood eosinophil count in the combined tralokinumab group $0.19 \pm 0.56$ cells $\times 10^{9} \cdot \mathrm{L}^{-1}$ compared to the placebo group $0.03 \pm 0.14$ cells $\times 10^{9} \cdot \mathrm{L}^{-1}$ at week 13 . Limited numbers of subjects had sputum cytology available. In the 24 subjects receiving tralokinumab, the geometric mean \pm geometric SD eosinophils was $2.9 \pm 6.55 \%$ at baseline and $6.2 \pm 4.55 \%$ at week 13 versus $3.5 \pm 5.7 \%$ at baseline and $2.6 \pm 5.7 \%$ at week 13 in the seven patients receiving placebo.

\section{Pharmacokinetics and immunogenicity}

The increase in tralokinumab exposure was approximately proportional to dose over the range and within each dose group serum concentrations increased approximately two-fold 

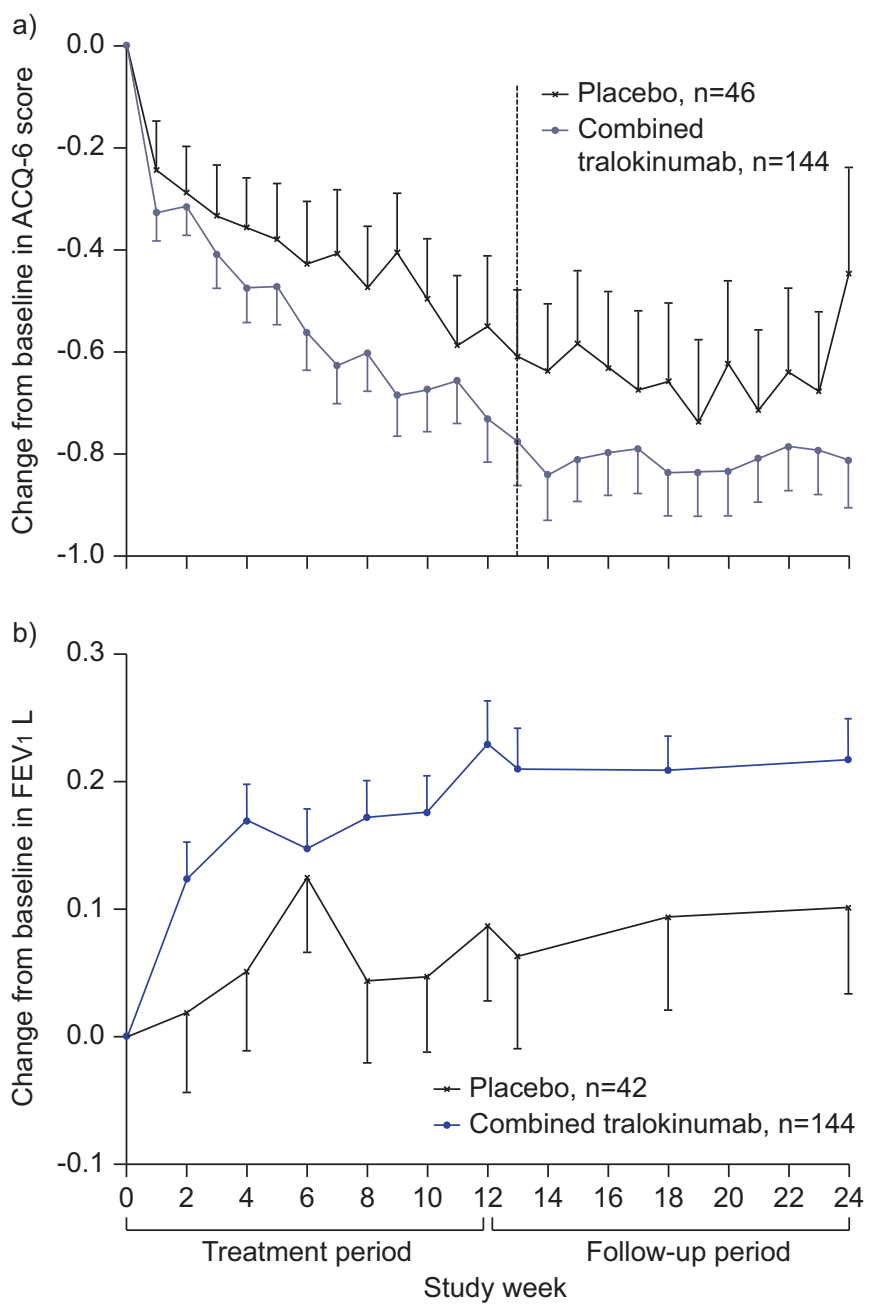

FIGURE 2. Mean ( $\mathrm{SE}$ ) change from baseline in a) Asthma Control Questionnaire, mean of six individual item scores (ACQ-6) score, and b) prebronchodilator forced expiratory volume in $1 \mathrm{~s}\left(\mathrm{FEV}_{1}\right)$ over time (evaluable population). ----: week 13, primary end-point.

between weeks 2 and 10 consistent with moderate accumulation. Comparable serum concentrations were observed at weeks 10 and 12, indicating that steady-state had been attained by week 10 . At all three doses evaluated plasma concentrations of tralokinumab were $>10$-fold lower at week 24 compared to week 12 . No serum anti-drug antibodies were detected during the course of the study.

\section{DISCUSSION}

A growing body of evidence implicates IL-13 as a key mediator in the development and maintenance of asthma. Tralokinumab is a human IgG4 monoclonal antibody which potently and specifically neutralises IL-13 [9]. This clinical study investigated the addition of tralokinumab to currently available asthma controller therapy in subjects with uncontrolled moderate-tosevere asthma. No impact was observed on ACQ-6 scores compared to placebo (primary end-point) but the increases in FEV1 and reduction in the use of short-acting $\beta_{2}$-agonists in tralokinumab-treated subjects are indicative of a treatment effect. The percentage increase in FEV1 from baseline ranged
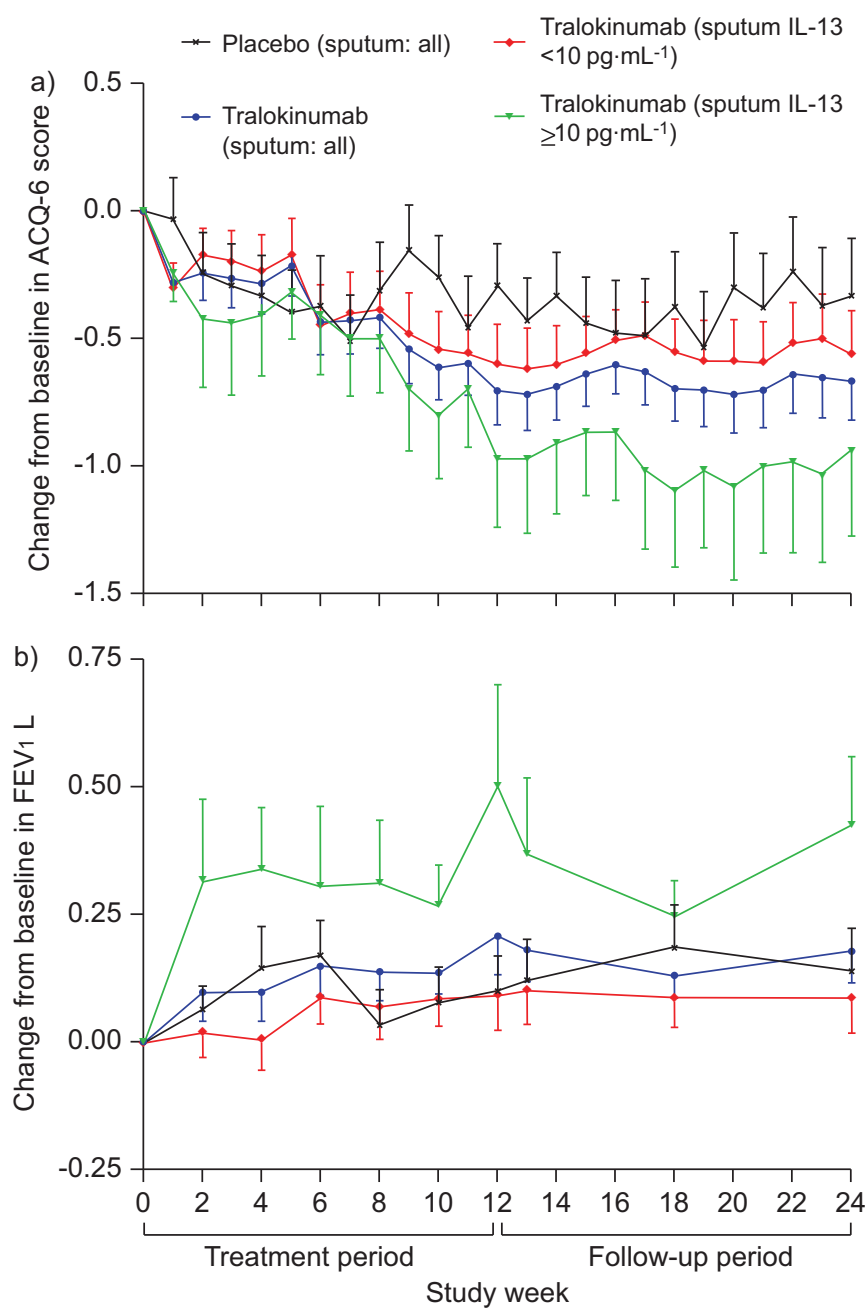

FIGURE 3. Mean (SE) change from baseline in a) Asthma Control Questionnaire, mean of six individual item scores (ACQ-6) score, and b) prebronchodilator forced expiratory volume in $1 \mathrm{~s}$ (FEV1) over time (evaluable population) in the subpopulation $(n=56)$ who supplied a sputum sample at baseline. Placebo (sputum: all) placebo: $n=17$; tralokinumab (sputum: all): $n=39$; tralokinumab-treated group without measurable IL-13 (sputum IL-13 $<10 \mathrm{pg} \cdot \mathrm{mL}^{-1}$; $\mathrm{n}=28$ ) and those with measurable $\mathrm{IL}-13$ (sputum IL-13 $\geqslant 10 \mathrm{pg} \cdot \mathrm{mL}^{-1} ; \mathrm{n}=11$ ).

between $8.1 \%(150 \mathrm{mg}$ ) and $16.1 \%$ (600 mg), which approximates the minimal important difference of $10 \%$ [14] and suggests that tralokinumab has the potential to deliver a clinically important response.

There was a marked placebo response in ACQ-6 starting during the 2-week screening period and continuing after randomisation. The placebo response has been well described in asthma studies and various explanations have been proposed, including suggestibility, natural disease variability, improved compliance with concomitant treatment and regression to the mean $[15,16]$.

It is possible that the principal treatment-related effect observed in our study, the improvement in pulmonary function, may reflect the role of IL-13 in the pathogenesis of asthma. A recently introduced concept is that asthma is heterogeneous, with different disease mechanisms driving particular asthma phenotypes 
TABLE 3 Adverse events reported overall and by $\geqslant 5 \%$ of subjects in any treatment group (safety population)

\begin{tabular}{|c|c|c|c|c|c|}
\hline \multirow[t]{2}{*}{ Adverse event } & \multirow[t]{2}{*}{ Placebo ${ }^{\#}$} & \multicolumn{4}{|c|}{ Tralokinumab } \\
\hline & & Combined tralokinumab & $150 \mathrm{mg}$ & $300 \mathrm{mg}$ & $600 \mathrm{mg}$ \\
\hline Subjects & 47 & 146 & 47 & 51 & 48 \\
\hline At least one adverse event & $17(36.2)$ & $70(47.9)$ & 20 (42.6) & $25(49.0)$ & $25(52.1)$ \\
\hline Asthma" & $3(6.4)$ & $16(11.0)$ & $5(10.6)$ & $5(9.8)$ & $6(12.5)$ \\
\hline Bacteriuria & $0(0.0)$ & $8(5.5)$ & $2(4.3)$ & $2(3.9)$ & $4(8.3)$ \\
\hline Decreased neutrophil count & $3(6.4)$ & $6(4.1)$ & $3(6.4)$ & $1(2.0)$ & $2(4.2)$ \\
\hline Influenza-like illness & $2(4.3)$ & $6(4.1)$ & $1(2.1)$ & $2(3.9)$ & $3(6.3)$ \\
\hline Urinary tract infection & $0(0.0)$ & $6(4.1)$ & $3(6.4)$ & $1(2.0)$ & $2(4.2)$ \\
\hline Crystalluria & $0(0.0)$ & $5(3.4)$ & $1(2.1)$ & $1(2.0)$ & $3(6.3)$ \\
\hline Diarrhoea & $0(0.0)$ & $5(3.4)$ & $0(0.0)$ & $0(0.0)$ & $5(10.4)$ \\
\hline
\end{tabular}

[17]. In pre-clinical models of asthma, IL-13 drives the variable airflow obstruction mechanism of AHR [5-7, 9], probably through direct effects on smooth muscle [17-19], but has only modest impact on eosinophilic inflammation [5, 9]. It is therefore plausible that in human asthma IL-13 neutralisation may predominantly impact variable airflow limitation mechanisms, resulting in the observed effects of tralokinumab on FEV1 and the absence of a reduction in sputum eosinophil counts, although the interpretation of this result must be treated with caution due to the small numbers of subjects with sputum cytology available. It is interesting to note that in our study the effect on FEV1 was observed in subjects with and without objective evidence of atopy; this is consistent with the previous finding that IL-13 mRNA is elevated in the bronchial mucosa of both atopic and non-atopic subjects with asthma [20].

Reports of other antibodies directed against IL-13 put the tralokinumab data into context. The addition of lebrikizumab, an anti-IL-13 IgG4 humanised monoclonal antibody, to existing controller therapies in a similar population has also been reported to be associated with improvements versus placebo in pre-bronchodilator FEV1, a trend towards a lower rate of protocol-defined exacerbations, but no impact on asthma symptoms as measured by ACQ-5 [21]. The IL-13-neutralising antibody IMA-638 inhibited the early and late airway responses to allergen challenge in mild atopic asthma patients [22]. These data, together with the results of our study, support the view that IL-13 may be an important mediator in asthma and that blockade of this cytokine may have the potential to provide a new therapeutic approach to the treatment of asthma. The apparent discordance between the treatment related improvement in pre-bronchodilator FEV1 and the absence of effect on ACQ score following IL-13 blockade by tralokinumab (ACQ-6) and lebrikizumab (ACQ-5) is of interest, and could be ascribed to either limitations in the sensitivity of the score, the large placebo effect observed, short trial duration, a selective mechanistic effect or indeed a combination of these factors. However, it is increasingly understood that asthma is a heterogeneous disease and therefore identification of potential subgroups or individual subject characteristics is likely to be key in delivering optimal response, with biotherapeutics that target specific mechanisms $[15,16,21]$. It is reasonable to postulate that subjects who have upregulated airway IL-13, as defined by a high T-helper cell type 2 signature [23], may be more likely to respond to IL-13-neutralising therapy. Elevated serum periostin levels have been identified as a potential surrogate measure of IL-13 activity and subgroup analyses of subjects with abovemedian baseline periostin levels who show larger increases in FEV1 than those with below-median baseline serum periostin following exposure to lebrikizumab [21] require replication. We explored this concept by examining 56 subjects with a baseline sputum sample. When subjects were stratified into sputum IL13-positive or -negative there was an apparent association between presence of IL-13 and response to tralokinumab (fig. 3). Taken together, these data suggest that if, in the future, subjects with upregulated lung IL-13 could be identified prospectively, then the clinical response to tralokinumab might be more clearly observed.

It is recognised that this proof-of-concept study has a number of limitations. First, the sample size was theoretically adequate to detect a difference in the change from baseline in mean ACQ- 6 score of 0.5 between combined tralokinumab groups and placebo; however, it did not account for potential heterogeneity with regard to the presence or absence of elevated levels of IL-13 in the lung within the study population, and it seems likely that the overall treatment effect observed was lowered by the presence of subjects who lacked the potential to respond to tralokinumab. In addition, the study was not fully powered to either compare the specific effects of individual tralokinumab 
doses over placebo or to determine the impact of tralokinumab on the frequency of asthma exacerbations. Other limitations include the short treatment duration, which is unlikely to have allowed the complete efficacy profile of tralokinumab to be established. For example, any treatment effect resulting from blockade of the putative pro-fibrotic effect of IL-13 is unlikely to be observed after 12 weeks dosing. Also, post-bronchodilator spirometry was not undertaken, and it is therefore not possible to confirm whether the treatment-related effect of tralokinumab on FEV1 is maintained in the presence of a $\beta_{2}$-agonist, though it should be noted that following treatment with lebrikizumab, a statistically significant effect on post-bronchodilator FEV1 was observed compared to placebo [21]. These issues will be specifically addressed in the future study of tralokinumab.

Tralokinumab demonstrated an acceptable safety profile with $94 \%$ of subjects receiving all scheduled doses. No serious AEs were considered to be associated with tralokinumab. The most frequent AEs reported were asthma, headache and nasopharyngitis. Some AEs, such as bacteriuria, urinary tract infections, crystalluria and diarrhoea were only observed among subjects treated with tralokinumab, although these did not result in either early withdrawal or sequelae; further studies will be necessary to determine whether these events are related to blockade of IL-13. Increased peripheral eosinophil count in the tralokinumab treatment group is consistent with that previously reported following exposure to lebrikizumab [21].

In summary, the addition of s.c. tralokinumab to existing asthma controller medication showed no significant improvement in ACQ-6 score. However, the observed effect of tralokinumab on FEV1 and other secondary end-points suggest that neutralisation of IL-13 may result in a therapeutic benefit in subjects with uncontrolled moderate-to-severe asthma. We conclude that this study provides evidence supporting the importance of IL-13 in asthma. Larger, longer studies are required to determine the potential role of tralokinumab in uncontrolled asthma and to identify those individuals most likely to demonstrate a clinical response.

\section{SUPPORT STATEMENT}

This study was funded by MedImmune.

\section{CLINICAL TRIAL}

This study is registered at Clinicaltrials.gov with identifier number NCT00873860.

\section{STATEMENT OF INTEREST}

Statements of interest for all authors and the study itself can be found at www.erj.ersjournals.com/site/misc/statements.xhtml

\section{ACKNOWLEDGEMENTS}

The authors thank R. Pereira (MedImmune LLC, Gaithersburg, MD, USA) for her critical review of the manuscript and her valuable comments; S. Fitzpatrick (MedImmune LLC) for assistance with interpretation of study safety data; the Inamed GmbH (Gauting, Germany) team for assistance with investigator site liaison; K. Kohlhase McLaurin (MedImmune LLC) for assistance with the operation and interpretation of the patient-reported outcome tools; and A. Herath (MedImmune Ltd, Cambridge, UK) for help with statistical interpretation. Medical writing and editorial assistance was provided by J. Stewart (QXV Communications, Macclesfield, UK).

\section{REFERENCES}

1 Guilbert TW, Garris C, Jhingran P, et al. Asthma that is not wellcontrolled is associated with increased healthcare utilization and decreased quality of life. J Asthma 2011; 48: 126-132.

2 Haselkorn T, Chen H, Miller DP, et al. Asthma control and activity limitations: insights from the Real-world Evaluation of Asthma Control and Treatment (REACT) study. Ann Allergy Asthma Immunol 2010; 104: 471-477.

3 Peters SP, Ferguson G, Deniz Y, et al. Uncontrolled asthma: a review of the prevalence, disease burden and options for treatment. Respir Med 2006; 100: 1139-1151.

4 Sullivan SD, Rasouliyan L, Russo PA, et al. Extent, patterns, and burden of uncontrolled disease in severe or difficult-to-treat asthma. Allergy 2007; 62: 126-133.

5 Wills-Karp M, Luyimbazi J, Xu X, et al. Interleukin-13: central mediator of allergic asthma. Science 1998; 282: 2258-2261.

6 Grunig G, Warnock M, Wakil AE, et al. Requirement for IL-13 independently of IL-4 in experimental asthma. Science 1998; 282: 2261-2263.

7 Zhu Z, Homer RJ, Wang Z, et al. Pulmonary expression of interleukin-13 causes inflammation, mucus hypersecretion, subepithelial fibrosis, physiologic abnormalities, and eotaxin production. J Clin Invest 1999; 103: 779-788.

8 Saha SK, Berry MA, Parker D, et al. Increased sputum and bronchial biopsy IL-13 expression in severe asthma. J Allergy Clin Immunol 2008; 121: 685-691.

9 May R, Monk P, Cohen E, et al. Preclinical development of CAT-354, an IL-13-neutralising antibody, for the treatment of severe uncontrolled asthma. Br J Pharmacol 2012; 166: 177-193.

10 Blanchard C, Mishra A, Saito-Akei H, et al. Inhibition of human interleukin-13-induced respiratory and oesophageal inflammation by anti-human-interleukin-13 antibody (CAT-354). Clin Exp Allergy 2005; 35: 1096-1103.

11 Singh D, Kane B, Molfino NA, et al. A phase 1 study evaluating the pharmacokinetics, safety and tolerability of repeat dosing with a human IL-13 antibody (CAT-354) in subjects with asthma. BMC Pulm Med 2010; 10: 3.

12 Juniper EF, O’Byrne PM, Guyatt GH, et al. Development and validation of a questionnaire to measure asthma control. Eur Respir J 1999; 14: 902-907.

13 Juniper EF, Bousquet J, Abetz L, et al. Identifying "wellcontrolled" and "not well-controlled" asthma using the Asthma Control Questionnaire. Respir Med 2006; 100: 616-621.

14 Reddel HK, Taylor DR, Bateman ED, et al. An official American Thoracic Society/European Respiratory Society statement: asthma control and exacerbations: standardizing endpoints for clinical asthma trials and clinical practice. Am J Respir Crit Care Med 2009; 180: 59-99.

15 Corren J, Busse W, Meltzer EO, et al. A randomized, controlled, phase 2 study of AMG 317, an IL-4R $\alpha$ antagonist, in patients with asthma. Am J Respir Crit Care Med 2010; 181: 788-796.

16 Bousquet J, Wenzel $S$, Holgate $S$, et al. Predicting response to omalizumab, an anti-IgE antibody, in patients with allergic asthma. Chest 2004; 125: 1378-1386.

17 Grunstein MM, Hakonarson H, Leiter J, et al. IL-13-dependent autocrine signaling mediates altered responsiveness of IgEsensitized airway smooth muscle. Am J Physiol Lung Cell Mol Physiol 2002; 282: L520-L528.

18 Tliba O, Deshpande D, Chen H, et al. IL-13 enhances agonistevoked calcium signals and contractile responses in airway smooth muscle. Br J Pharmacol 2003; 140: 1159-1162.

19 Deshpande DA, Dogan S, Walseth TF, et al. Modulation of calcium signaling by interleukin-13 in human airway smooth muscle: role of CD38/cyclic adenosine diphosphate ribose pathway. Am J Respir Cell Mol Biol 2004; 31: 36-42. 
20 Humbert M, Durham SR, Kimmitt P, et al. Elevated expression of messenger ribonucleic acid encoding IL-13 in the bronchial mucosa of atopic and nonatopic subjects with asthma. J Allergy Clin Immunol 1997; 99: 657-665.

21 Corren J, Lemanske RF, Hanania NA, et al. Lebrikizumab treatment in adults with asthma. N Engl J Med 2011; 365: 1088-1098.
22 Gauvreau GM, Boulet LP, Cockcroft DW, et al. Effects of interleukin-13 blockade on allergen-induced airway responses in mild atopic asthma. Am J Respir Crit Care Med 2011; 183: 1007-1014.

23 Woodruff PG, Modrek B, Choy DF, et al. T-helper type 2-driven inflammation defines major subphenotypes of asthma. Am J Respir Crit Care Med 2009; 180: 388-395. 